\title{
ENDOMETRIAL STUDY OF ABNORMAL UTERINE BLEEDING IN WOMEN AT 45 YEARS AND ABOVE
}

\section{ABSTRACT}

The study is done to know the causes for the abnormal uterine bleeding in perimenopausal women at Chitwan valley and to compare the histopathological findings between the pre-menopausal and postmenopausal women. Retrospective analysis of patients who have had abnormal bleeding during a period of one and a half years' from January 2000 to June 2001 in Department of Gynaecological Oncology of BP Koirala Memorial Cancer Hospital (BPKMCH), Chitwan, Nepal. There were total sixty women (aged 45 years and above) having abnormal bleeding managed at BPKMCH. A total of sixty patients were studied. Majority (57\%) of them was postmenopausal ladies presented with abnormal vaginal bleeding. The age range was 45 to 81 years. Only $37 \%$ had pathological bleeding including malignancies and only about $17 \%$ had malignancies. Malignant diseases were found in $7.7 \%$ cases in the pre-menopausal ladies whereas it was $\mathbf{2 3 . 4 \%}$, almost three times higher, in the post-menopausal ladies. Carcinoma of the cervix and of the endometrium occurred with increasing frequency with increasing age in this study. So, a thorough work-up is needed for the elderly women presenting with an abnormal vaginal bleeding especially to rule out malignancies.

Key Words: endometrial biopsy, endometrial cancer, postmenopausal bleeding.

\section{INTRODUCTION}

Abnormal uterine bleeding is one of the most common problems that challenge the gynecologists. And it is one of the commonest symptoms of reproductive system disorder. These patients present to a clinician with abnormal per vaginal bleeding.

The abnormal bleeding can be caused by a wide variety of disorders. It may represent a normal physiological state, and observation alone may be warranted. Alternatively, the bleeding can be a sign of a serious underlying condition necessitating aggressive treatment that could include a major procedure. ${ }^{1}$

The causes for the bleeding in elderly women are hormonal, pregnancy complications, bleeding diathesis and more importantly local pathology including malignancy, benign tumors, and infection. Although the bleeding in the elderly in perimenopausal period may be secondary to estrogen withdrawal (physiological state) otherwise known as Dysfunctional Uterine Bleeding (DUB), the incidence of structural pathology increases with the advancing age. ${ }^{2}$ In some cases it may be due to malignancy of the reproductive organs, particularly in postmenopausal women.

Address for correspondence : Dr. Ganesh Dangal

Dept. of Obstetrics and Gynaecology

Kathmandu University Medical School, Banepa

Email: gareshma@hotmail.com 
The need to embark upon a diagnostic curettage in perimenopausal women cannot be overemphasized. ${ }^{2}$ It is now generally accepted that an adequate clinical examination of abdomen and pelvis, and uterine curettage, hysteroscopy or at least and endometrial biopsy are essential to exclude organic disease of the uterus in these women. ${ }^{3}$

The present study was carried out firstly to find out the causes for abnormal uterine bleeding in Nepalese perimenopausal women at Chitwan valley and secondly to compare the histopathological findings between the pre-menopausal and postmenopausal women.

\section{PATIENTS AND METHODS}

The study population comprised of women aged 45 year and above who attended the gynecological oncology outpatient's department at B.P. Koirala Memorial Cancer Hospital (BPKMCH), Bharatpur, from January 2000 to June 2001. A total of 60 consecutive patients who underwent examination under anesthesia (EUA), dilatation and curettage (D\&C) and endometrial biopsy for diagnostic reasons were included in the study.
Each had abnormal uterine bleeding and all the patients with abnormal vaginal bleeding, except those with pregnancy complications and clinically diagnosed cases of local lesions like cancer of the cervix, were included in the study.

All procedures were performed under intravenous anesthesia. Details of the histopathological reports were obtained from the laboratory.

\section{RESULTS}

Sixty women were included in the study. Of these 34(57\%) were postmenopausal and 26(43\%) were premenopausal. Their age ranged from 45 to 81 years with a mean age of 63 years. All were multiparous housewives and $16 \%$ had positive smoking history.

Of all the patients with the bleeding, only 22 patients (37\%) had pathological bleeding and ten patients ( $17 \%$ of all patients) had malignant diseases as shown in Table I.

Among the 60 patients, majority (22 patients i.e.37\%) had 